\title{
High sensitivity magnetometer for measuring the isotropic and anisotropic magnetisation of small samples
}

\author{
A. McCollam, a) P. G. van Rhee, J. Rook, E. Kampert, U. Zeitler, and J. C. Maan \\ High Field Magnet Laboratory, Institute of Molecules and Materials, Radboud University Nijmegen, \\ Toernooiveld 7, 6525 ED Nijmegen, The Netherlands
}

(Received 17 January 2011; accepted 8 May 2011; published online 31 May 2011)

\begin{abstract}
We describe how the full, isotropic and anisotropic, magnetisation of samples as small as tens of micrometers in size can be sensitively measured using a piezoresistive microcantilever and a small, moveable ferromagnet. Depending on the position of the ferromagnet, a strong but highly local field gradient of up to $\sim 4200 \mathrm{~T} / \mathrm{m}$ can be applied at the sample or removed completely during a single measurement. In this way, the magnetic force and torque on the sample can be independently determined without moving the sample or cycling the experimental system. The technique can be used from millikelvin temperatures to $\sim 85 \mathrm{~K}$ and in magnetic fields from $2 \mathrm{~T}$ to the highest fields available. We demonstrate its application in measurements of the semimagnetic semiconductor $\mathrm{Hg}_{1-x} \mathrm{Fe}_{x} \mathrm{Se}$, where we achieved a moment sensitivity of better than $2.5 \times 10^{-14} \mathrm{~J} / \mathrm{T}$ for both isotropic and anisotropic components. (C) 2011 American Institute of Physics. [doi:10.1063/1.3595676]
\end{abstract}

\section{INTRODUCTION}

Magnetometry is one of the most fundamental and versatile techniques for the study of condensed matter systems. Investigations of characteristic properties such as magnetic order or interactions, phase transitions, superconductivity, or the details of conduction electrons at the Fermi surface, frequently rely on sensitive measurements of static or oscillatory magnetisation. ${ }^{1}$

A large number of methods have been developed to measure magnetisation, with sensitivities and applicability that vary so widely with experimental conditions and sample type that it is not generally instructive to discuss a single figure of merit: experimental criteria, such as high magnetic fields or low temperatures, tend to dictate both the optimal method and the maximum achievable sensitivity. For example, SQUID and vibrating sample magnetometers, Hall sensors, torque magnetometers, or force methods are used preferentially in different research areas to characterise the magnetic properties of such diverse systems as biomedical labels, high temperature superconductors, or the submicron probe tips required for magnetic resonance force microscopy (MRFM). ${ }^{2-8}$

Despite the variety of techniques available, it is experimentally challenging to measure all desirable aspects of the magnetisation in a simple and unified way on a single sample. Torque methods are most commonly employed to measure anisotropic magnetisation and have the advantage of very high sensitivity across a wide dynamic range in magnetic field, temperature and sample size: ${ }^{9-11}$ moment sensitivity of $3 \times 10^{-17} \mathrm{~J} / \mathrm{T}$ has been reported for nanometer sized samples integrated into micromechanical cantilevers, ${ }^{12}$ and $\left(10^{-13}-10^{-14}\right) \mathrm{J} / \mathrm{T}$ for bulk samples on several different types of torquemeter. ${ }^{6,9,13,14}$ To measure isotropic magnetisation in a similarly wide range of experimental conditions is more difficult, due to the limitations of many techniques at high magnetic fields or low temperatures: $2,4,15$

a)Electronic mail: A.McCollam@science.ru.nl. sensitivity of $10^{-19} \mathrm{~J} / \mathrm{T}$ has been reported using dynamicmode cantilever force magnetometry, ${ }^{16}$ but this resolution is specific to submicron sized ferromagnetic samples, and cannot, at present, be translated to bulk or non(ferro)magnetic samples, for which isotropic moment sensitivities are reduced to $\sim 10^{-12} \mathrm{~J} / \mathrm{T}$ or lower. ${ }^{17}$ Measuring both anisotropic and isotropic components of magnetisation, therefore, normally involves not only changing the method of measurement, for example, from a torque to a force method, or changing the position of the experiment inside a magnet coil, ${ }^{17,18}$ but also a significantly lower sensitivity for the isotropic component. If measurements of magnetic quantum oscillations are desired, the need for high sensitivities becomes even more stringent, and often cannot be met by non-specialised apparatus. Additional difficulties related to sensitivity can arise for bulk samples when they are very small (e.g., a few micrograms).

Here we report a type of torque magnetometer, which allows us to measure the full isotropic, anisotropic, and oscillatory magnetisation of a small bulk sample with very high sensitivity, in a simple and convenient way without moving the sample or modifying the experimental setup.

To demonstrate the applicability and sensitivity of our technique, we describe measurements on the semimagnetic semiconductor $\mathrm{Hg}_{1-x} \mathrm{Fe}_{x} \mathrm{Se}$, which shows isotropic paramagnetism, and de Haas-van Alphen oscillations associated with a weakly anisotropic Fermi surface.

\section{PRINCIPLE OF THE TECHNIQUE}

Quite generally, a material with magnetisation $\mathbf{M}$, in a magnetic field $\mathbf{B}$, will experience a torque

$$
\tau=\nabla(\mathbf{M} \cdot \mathbf{B}) \times \mathbf{d}+\mathbf{M} \times \mathbf{B},
$$

where $\mathbf{d}$ is the distance from the sample to the torque axis. The first term arises from the isotropic magnetisation of the sample and leads to a force on the sample in a magnetic field gradient. The second term is due to the anisotropic 
magnetisation and depends on the component of $\mathbf{M}$ perpendicular to the applied field direction.

The technique we present here measures the total torque (1) experienced by a sample in a magnetic field applied perpendicular to the torque axis. To separate the terms in expression (1) and determine the isotropic and anisotropic components of magnetisation, each experiment is conducted in two parts: the first in a homogeneous magnetic field, giving the pure torque signal $\mathbf{M} \times \mathbf{B}$; the second in the presence of a field gradient, giving the sum of force and torque terms. Subtracting the first of these quantities from the second then gives the force term $\nabla(\mathbf{M} \cdot \mathbf{B}) \times \mathbf{d}$.

To measure the torque, we use a piezoresistive microcantilever. These devices have extremely high sensitivity ${ }^{6}$ and are widely used for measurements of de Haas-van Alphen oscillations or other relatively weak anisotropic magnetic signatures. ${ }^{11}$ Use of these cantilevers to measure isotropic magnetisation by force magnetometry has not, however, been previously reported.

The miniature size of the cantilevers means that platelet samples of dimensions $\sim 200 \times 200 \times 50 \mu \mathrm{m}^{3}$ are optimal, but the total moment of such a small sample is typically far too small to yield a measurable signal in available magnetic field gradients, i.e., $\nabla(\mathbf{M} \cdot \mathbf{B}) \times \mathbf{d}$ is negligible. The significant innovation in our technique is the very large magnetic field gradient we achieve by moving a small, strong ferromagnet close to the sample. This method of achieving a large field gradient is similar to that used in MRFM, where a ferromagnetic particle creates an inhomogeneous field which acts on the spins in the sample. ${ }^{19,20}$ Our magnetometer, however, creates a magnetic field gradient of sufficient strength and appropriate spatial distribution to create measurable forces on bulk samples with volume of the order $10^{-3} \mathrm{~mm}^{3}$ and typical mass of a few micrograms. We are, therefore, able to use the most sensitive microcantilever techniques available for such samples to make torque measurements of anisotropic magnetisation and force measurements of isotropic magnetisation, with comparable sensitivity, on the same sample during the same experiment. This significantly improves the sensitivity to isotropic magnetisation that is possible in measurements over a wide magnetic field and temperature range, and makes high sensitivity measurements of both the anisotropic and isotropic magnetisation of a small sample considerably easier and faster than they have been to date.

In the most general case, a ferromagnetic bar enhances the magnetic field in its vicinity by an amount $A(x, y, z) M_{0}$, where $M_{0}$ is the total magnetic moment of the bar, and $A$ depends on the dimensions of the bar and decreases rapidly over distances $x, y, z$ comparable to these dimensions. The field gradient produced at the surface of the bar can, therefore, be very large if its dimensions are small enough. ${ }^{21}$ For example, a gradient of $\sim 5000 \mathrm{~T} / \mathrm{m}$ can be generated at the surface of a $\mathrm{mm}$-sized bar of saturation magnetisation $\mu_{0} M=2.5 \mathrm{~T}$.

Our magnetometer incorporates a bar of ferromagnetic dysprosium, with dimensions $1.0 \times 0.5 \times 0.5 \mathrm{~mm}^{3}$. Figure 1(a) shows a sketch of this bar (inset) and the field gradient $d B / d x$ produced outside its lower surface, along the central axis $(y, z=0)$, for a total moment $M_{0}=5$ $\times 10^{-4} \mathrm{~J} / \mathrm{T}$. The small size of the sample on a piezo cantilever
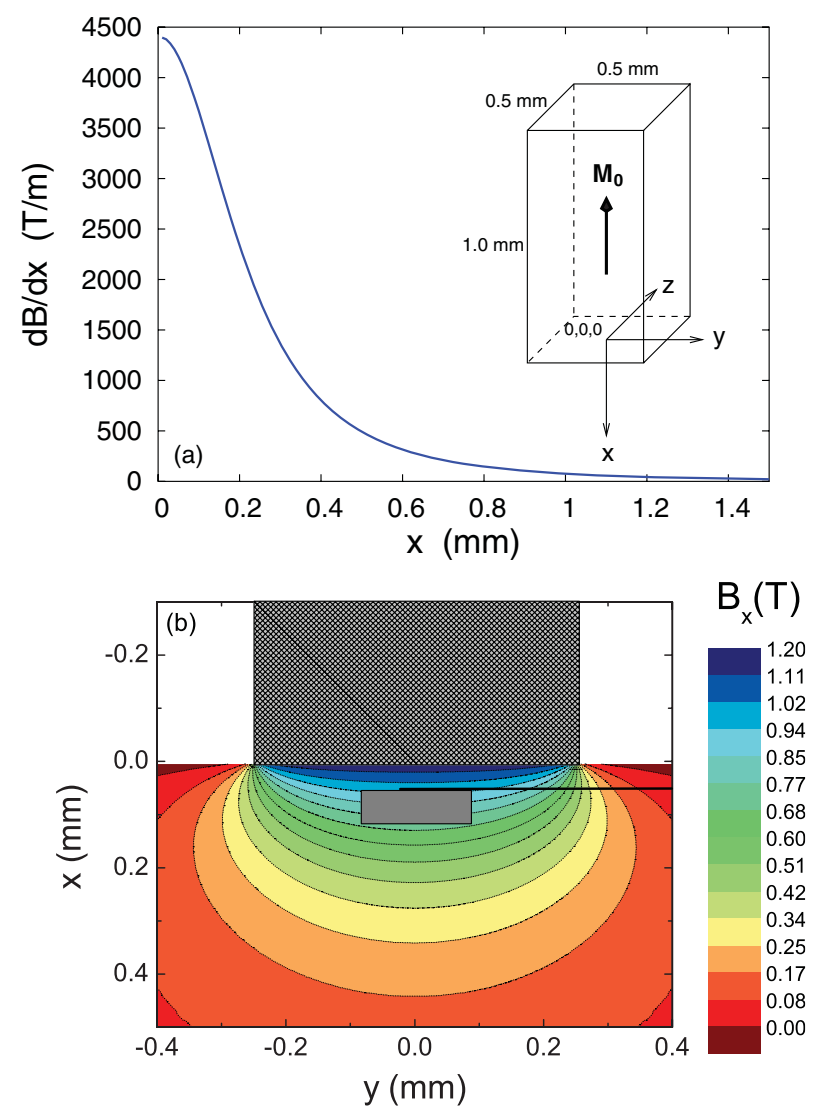

FIG. 1. (Color online) (a) (Inset) Sketch of the ferromagnetic bar discussed in the text. (Main figure) Magnetic field gradient produced as a function of distance $x$ below the bar, when $y, z=0$, and $M_{0}=5 \times 10^{-4} \mathrm{~J} / \mathrm{T}$. (b) Schematic of the same ferromagnetic bar (hatched grey) mounted close to the cantilever and sample (plain grey, side view), with a plot of the field profile below the bar.

makes it possible to position the ferromagnet very close to the sample $(<50 \mu \mathrm{m})$, so that the whole sample is in the region of highest and most uniform field gradient. Figure 1(b) gives a schematic view of this situation, with the lower part of the ferromagnet (hatched grey) and the sample on the cantilever (plain grey) shown against a colour representation of the magnetic field profile below the bar. ${ }^{22-24}$ The field profile is symmetric in the $y$ and $z$ directions, so the ferromagnet is positioned centrally above the sample. The size and geometry of the ferromagnet is such that for typical samples, with $x$ dimension $\simeq 50 \mu \mathrm{m}$, the field gradient is approximately uniform over the sample in the $y$ and $z$ directions, with variation of $d B / d x$ over the thickness of the sample that is small enough to have no obvious effect on experimental results. ${ }^{22,25}$

Our technique involves mounting the ferromagnet such that it can be moved between two fixed positions, close to (as in Fig. 1) or far from the sample on the cantilever. In this way, the field gradient can be applied at the sample or removed as required, and measurement of the isotropic and anisotropic components of the magnetisation can be carried out as described above.

The arrangement of cantilever, sample, and ferromagnet is extremely compact and fits easily into the limited space available in the bore of a high field magnet, such as our 33 T Bitter magnets at the HFML, Nijmegen. The amount of 
magnetic material present $\left(2.5 \times 10^{-4} \mathrm{~cm}^{3}\right.$ of dysprosium $)$ is small enough so that the magnetic forces involved are only a few tens of milliNewtons at high field and can easily be accommodated by the mount and supports on the magnetometer insert.

Creating a field gradient with a moveable ferromagnet has the considerable additional advantage that the sample can always be positioned in the field centre, in the maximum applied homogeneous field, so that measurements of isotropic and anisotropic magnetisation can both be carried out in the highest available applied field. This is in contrast to the more common situation, where measuring the isotropic signal involves moving the sample away from the centre of the field in order to make use of the natural field gradient of the magnet coil. ${ }^{17,18}$ Moreover, although it is often desirable to perform magnetisation measurements in the highest possible magnetic fields, because our technique only requires a field higher than the saturation field of the ferromagnet, it can be equally well employed in superconducting and lower field magnets.

\section{DESIGN OF THE MAGNETOMETER}

A detailed image of our magnetometer is shown in Fig. 2(a). It is currently incorporated into a ${ }^{3} \mathrm{He}$ cryogenic system and can be used at temperatures as low as $320 \mathrm{mK}$. The essential features of the magnetometer are labelled in the figure.

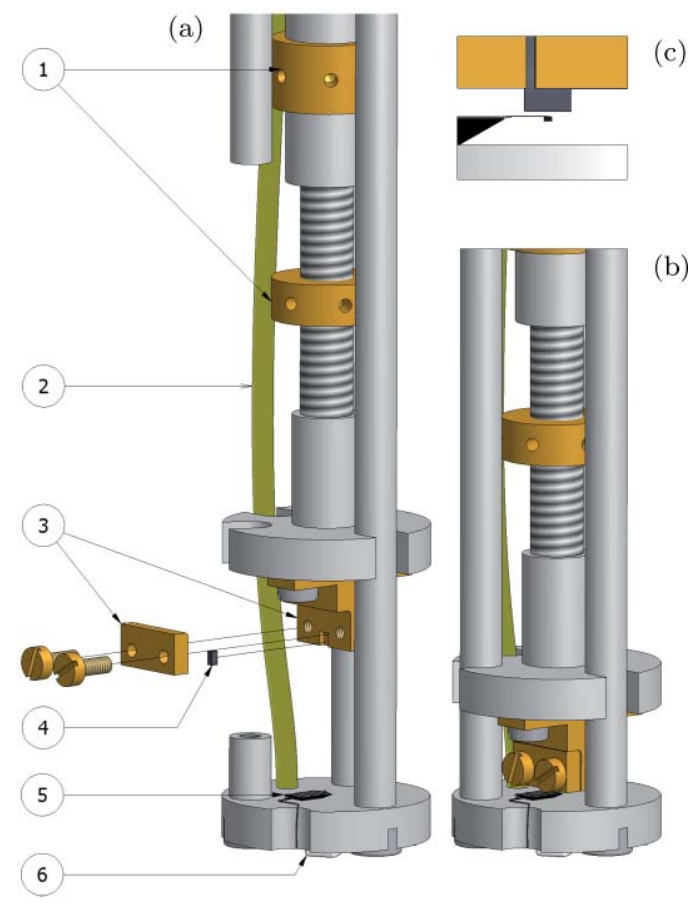

FIG. 2. (Color online) (a) (Magnetometer insert) Details of operation are discussed in the text. One of three upright supports is cut away for greater clarity. (1) Nuts for adjustment of differential screw (upper threads are not shown), (2) Electrical wiring, (3) Mount and clamp for ferromagnet, (4) Dysprosium bar, (5) Cantilever mounted on chip, (6) Heater and thermometer. (b) Insert with the upper platform fully lowered so that the ferromagnet is close to the sample. (c) Magnified image of the ferromagnet positioned close to the sample on the cantilever.
At the bottom of the insert is a fixed platform, on which the cantilever and sample are mounted (a $300 \Omega$ strain gauge heater and a ruthenium oxide thermometer for temperature control and measurement are mounted on the underside of this platform, as indicated). The ferromagnet is clamped on the upper platform, which can travel vertically over a distance of $1.5 \mathrm{~cm}$. We have used a simple lever at the top of the insert to control this motion: apart from its mechanical simplicity, this has the advantage of moving the upper platform between two fixed positions which are perfectly reproducible. Figures 2(a) and 2(b) illustrate the two possible positions of the upper platform. No lateral motion is possible, so the sample and ferromagnet are positioned in the centres of their respective platforms.

A particularly important feature of the magnetometer is the mechanism for fine-tuning the position of the ferromagnet with respect to the sample. Once the sample is mounted on the cantilever with the ferromagnet in its lower position close to the sample (Fig. 2(b)), precise position adjustment is made via a differential screw arrangement. Screw pitches of M3 $\times 0.5$ and M4 $\times 0.7$ give a vertical travel of $200 \mu \mathrm{m}$ per full turn. The ferromagnet-sample separation is measured using an eyepiece scale on an optical microscope and can be adjusted with a precision of a few microns. To avoid differential thermal contraction, the body of the magnetometer and all supports were made from the same type 304 stainless steel. Figure 2(c) shows an example of the final position of the ferromagnet and sample. Figures 1(a) and 1(b) illustrate the need to make the ferromagnet-sample separation as small as possible, as the field gradient falls away steeply with increasing distance from the edge of the ferromagnet. During a typical measurement, we expect the maximum deflection of the cantilever to be less than $5 \mu \mathrm{m}$, but the limitation on the ferromagnetsample separation is rather determined by the precision that can be obtained when making adjustments under a standard optical microscope. For the work described here, this separation was $30 \mu \mathrm{m}$, unless otherwise stated. Once this separation is fixed, the upper platform and ferromagnet can be moved $1.5 \mathrm{~cm}$ away and reproducibly returned to this position as desired. Repeated measurements showed this position to be reproducible within the noise limits of the data shown in Figs. 4 and 5. As the field gradient due to the ferromagnet falls to zero at a distance of about $1.5 \mathrm{~mm}$ from the surface of the ferromagnet, it has no influence on the sample at a distance of $1.5 \mathrm{~cm}$.

The size of the field gradient produced by the ferromagnet depends on its geometry and strength. We used dysprosium (Dy) for our ferromagnetic material, due to its extremely high saturation induction of $\sim 3.6 \mathrm{~T}^{26,27}$ The bar of $0.5 \times 0.5 \times 1.0 \mathrm{~mm}^{3}$ was made by clamping together five $0.5 \times 1.0 \mathrm{~mm}^{2}$ pieces of $0.1 \mathrm{~mm}$ thick Dy foil. ${ }^{28}$ The bottom surface of the bar, the surface closest to the sample, was polished to ensure that it was perfectly flat. A photograph of the Dy magnet used is shown in inset (i) of Fig. 3. It is held in a brass clamp, as is also shown schematically in Fig. 2.

The magnetisation of the Dy foil was measured independently using an extraction magnetometer and the resulting curve is plotted in Fig. 3. The figure shows that the 


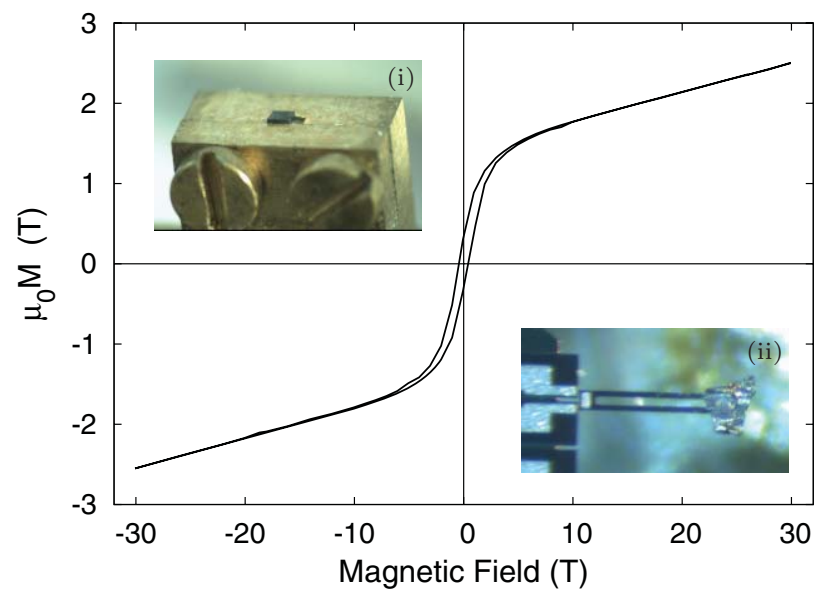

FIG. 3. (Color online) Magnetisation of Dy foil used to make our ferromagnetic bar. (i) Photograph of Dy magnet. (ii) Photograph of Seiko PRC400 piezo-microcantilever with sample A1.

magnetisation does not saturate up to $30 \mathrm{~T}$ and reaches only $70 \%$ of its potential maximum value. This is probably due to the polycrystalline nature of the foil and the large magnetocrystalline anisotropy of Dy. The resulting magnetic field gradient at the sample, however, was still large enough for our purposes. For the maximum Dy magnetisation $\left(\mu_{0} M=2.5 \mathrm{~T}\right.$ obtained at an applied field of $30 \mathrm{~T}$, as shown in Fig. 3), the maximum field gradient at a distance of $30 \mu \mathrm{m}$ from the ferromagnet, was $\sim 4200 \mathrm{~T} / \mathrm{m}$.

The piezoresistive cantilever we used for this work is commercially available from Seiko Instruments. ${ }^{29,30} \mathrm{~A}$ piezoresistive path runs along the lever and the stress caused by deflection of the lever creates a proportional change in the resistance of this path, which is detected via a sensing current. The cantilever is embedded on a chip, which also holds a "dummy" lever, with an identical piezoresistive path. The resistance of both levers was measured as part of a Wheatstone bridge circuit, using an ac excitation across the bridge and lock-in measurement techniques to allow sensitive detection of small resistance changes. In this configuration, the dummy lever compensates resistance changes not due to deflection of the sample. Figure 3(ii) shows a photograph of the cantilever with sample A1 used during this work. The lever has dimensions $400 \times 50 \times 5 \mu \mathrm{m}^{3}$ and a spring constant in the range $2-4 \mathrm{~N} / \mathrm{m}^{30}$ The lever length of $400 \mu \mathrm{m}$ proved to be crucial to the success of our method: the isotropic torque signal is directly proportional to the length of the cantilever, and we found that using a shorter cantilever of length $120 \mu \mathrm{m}$ gave greatly inferior results.

We note that the Curie temperature of dysprosium is $85 \mathrm{~K}$ and sets the upper limit for the operation temperature of the magnetometer. ${ }^{31}$

\section{EXPERIMENTAL RESULTS}

To test the operation and sensitivity of our magnetometer, we carried out a number of measurements on the semimagnetic semiconductor $\mathrm{Hg}_{1-x} \mathrm{Fe}_{x} \mathrm{Se}$. The samples used contain Fe ions in the $3^{+}$valence state only, and the magnetisation of the system comprises the isotropic Brillouin paramagnetism of $\mathrm{Fe}^{3+}$, the de Haas-van Alphen effect of free electrons on a weakly anisotropic Fermi surface, and an isotropic background diamagnetism from the $\mathrm{HgSe}$ lattice. ${ }^{18}$ We thus expect the torque signals to be

$$
\begin{aligned}
\tau_{\text {anisotropic }} & =\tilde{\tau}_{a}, \\
\tau_{\text {isotropic }} & =\tau_{\text {lat }}+\tau_{B}+\tilde{\tau}_{i},
\end{aligned}
$$

where $\tau_{\text {lat }}$ is the background signal due to the lattice, $\tau_{B}$ is the paramagnetic Brillouin contribution, and $\tilde{\tau}_{a}$ and $\tilde{\tau}_{i}$ are the oscillatory torques due to the anisotropic and isotropic components of the de Haas-van Alphen (dHvA) effect, respectively. As we will show, we can clearly observe all these contributions in our data.

We performed measurements on two small samples cut from a large oriented single crystal (sample A in Ref. 18). These samples were slightly different in size and were mounted on the cantilever with different orientations relative to the applied magnetic field direction: The first sample, A1 in the following, was a slightly irregular platelet (Fig. 3(b)) with approximate dimensions $150 \times 100 \times 50 \mu \mathrm{m}^{3}$, and was oriented with the magnetic field direction approximately $25^{\circ}$ from the [001] crystallographic axis, towards [110]; the second sample, A2, had dimensions $\sim 80 \times 80 \times 50 \mu \mathrm{m}^{3}$ and was mounted with the field parallel to [001] (within $3^{\circ}$ ).

The measurements reported here were carried out at a temperature of $1.3 \mathrm{~K}$, a magnetic field sweep rate of $1 \mathrm{~T} / \mathrm{min}$ and with a sensing current of $100 \mu \mathrm{A}$ to the cantilever. All results and calculations have been corrected for the enhancement of the magnetic field at the sample when the dysprosium ferromagnet is close by.

Figure 4 shows the torque data from sample A1. The main Fig. 4(a) shows raw data taken with and without the applied magnetic field gradient, $\tau_{\nabla B}$ and $\tau_{\nabla B=0}$, respectively. With $\nabla B=0$, the signal increases roughly linearly with field and shows a weak oscillatory structure. The only torque

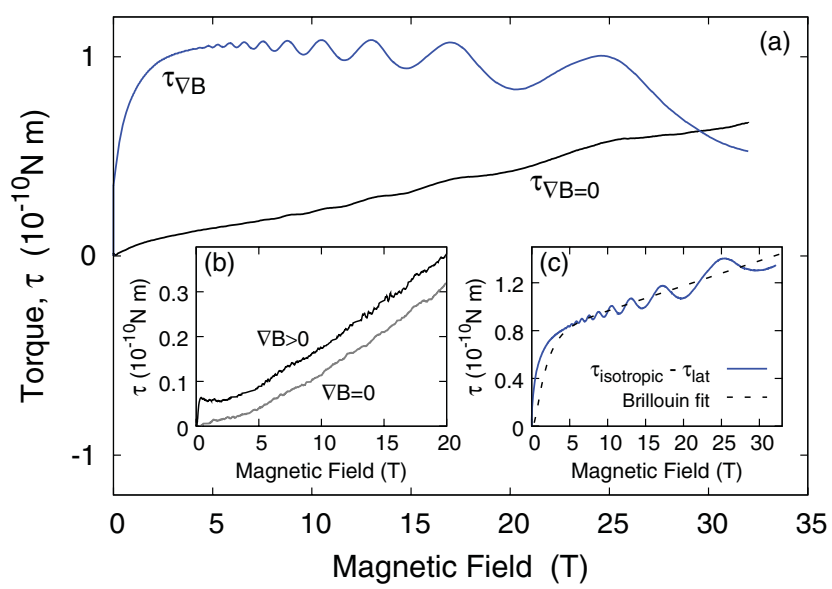

FIG. 4. (Color online) (a) Raw torque data from sample A1; $\tau_{\nabla B=0}$ corresponds to the $\mathbf{M} \times \mathbf{B}$ term in Eq. (1), plus any background from the cantilever. (b) Torque signal with and without a field gradient from an empty cantilever. This lever was shorter than that used for the $\mathrm{Hg}_{1-x} \mathrm{Fe}_{x}$ Se measurements $(120 \mu \mathrm{m}$ rather than $400 \mu \mathrm{m})$, but the devices are otherwise identical and their responses to a magnetic field and gradient are similar. (c) Isotropic signal showing Brillouin and oscillatory components. The cantilever and lattice backgrounds have been removed. 
signal we expect in this configuration is the anisotropic dHvA effect, so we can take the approximately field-linear background to be characteristic of the cantilever. Measurements in the absence of a sample (inset (b) of Fig. 4) produce a similar signal which, apart from a sharp jump when the field is initially switched on, is unaffected by the presence of a field gradient. Figure 4(b) is given as torque vs magnetic field, but this signal from the empty cantilever is probably magnetoresistive in origin, and appears because the Wheatstone bridge is imperfectly balanced.

When we apply a field gradient $\nabla B$, a strikingly different signal is observed. Subtracting the lower from the upper curve $\left(\tau_{\nabla B}-\tau_{\nabla B=0}\right)$ removes the anisotropic contribution and the background due to the cantilever and gives $\tau_{\text {isotropic }}$. Inset (c) of Fig. 4, shows this signal with the diamagnetic lattice background also subtracted and demonstrates how the Brillouin paramagnetism of the $\mathrm{Fe}^{3+}$ ions and the isotropic dHvA oscillations can be extracted. The dotted line in Fig. 4(b) is the theoretically expected Brillouin curve for an $\mathrm{Fe}^{3+}$ concentration of $2.35 \times 10^{18} \mathrm{~cm}^{-3}$, which is the value inferred from the observed dHvA frequency, assuming parabolic bands and the conventional theoretical relations. The number of free electrons contributing to the $\mathrm{dHvA}$ effect and the number of $\mathrm{Fe}^{3+}$ ions are expected to be equal, and the experimentally determined value is in excellent agreement with the nominal doping concentration of $2.5 \times 10^{18} \mathrm{~cm}^{-3}$. The slight departure of the experimental data from the theoretical Brillouin curve at very low field is due to an initial sharp jump in the torque signal in the presence of the field gradient. A similar jump is seen in the response of the empty lever (Fig. 4(b)) and is probably related to the rapidly increasing magnetisation of Dy in this field region (Fig. 3).
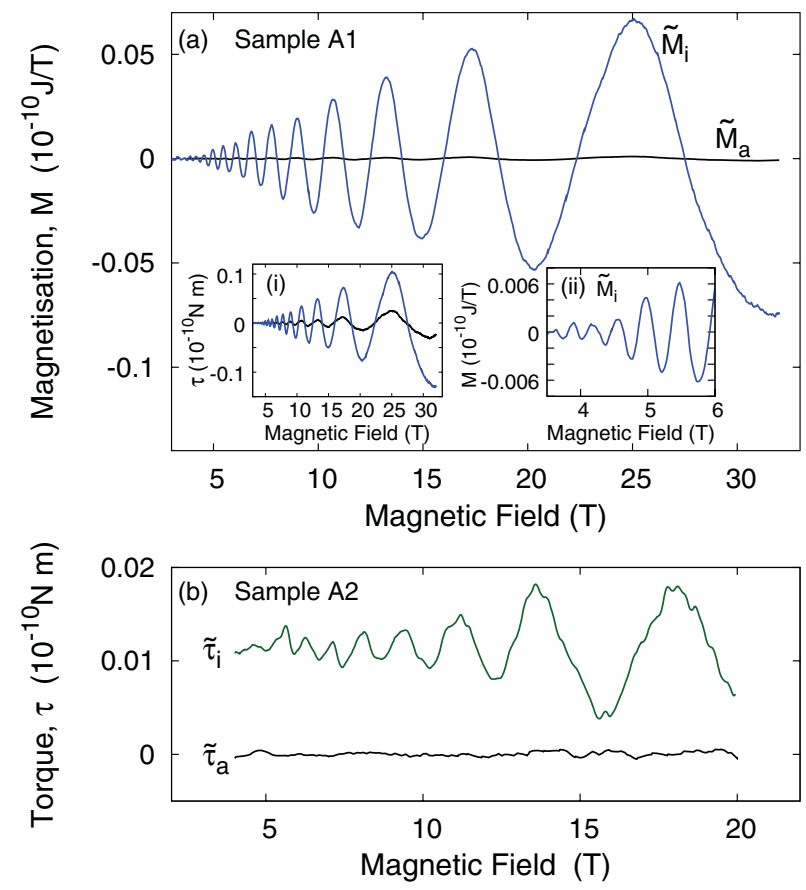

FIG. 5. (Color online) (a) Oscillatory magnetisation of sample A1. Inset (i) shows the same data plotted as torque. Inset (ii) is an expanded view of the low field magnetisation oscillations in the main figure. (b) Torque oscillations from sample A2.
Figure 5(a) (main panel) shows the oscillatory components of the isotropic and anisotropic magnetisation of sample A1. Inset (i) shows the corresponding torque plot. The oscillations are periodic in inverse magnetic field and can be well described by the Lifshitz-Kosevich theory of the dHvA effect, ${ }^{32}$ with a single frequency of $55.69 \mathrm{~T}$. The oscillation freqency is approximately the same for $\tilde{M}_{i}$ and $\tilde{M}_{a}$, which reflects the very weak anisotropy of the $\mathrm{Hg}_{1-x} \mathrm{Fe}_{x}$ Se Fermi surface. Inset (ii) of Fig. 5(a) is an expanded view of $\tilde{M}_{i}$ at low field; oscillations of amplitude $\sim 4 \times 10^{-14} \mathrm{~J} / \mathrm{T}$ are clearly resolvable and illustrate the high sensitivity of our force measurement.

Figure 5(b) shows torque data from sample A2, with background and Brillouin contributions removed. This sample is mounted with the applied magnetic field parallel to a primary crystallographic direction and, as expected for this orientation, no $\mathrm{dHvA}$ oscillations are resolvable in the anisotropic response. Clear oscillations, however, were measured in the isotropic signal $\tilde{\tau}_{i}$.

Figure 5 illustrates an important aspect of our results. The huge difference in the amplitudes of $\tilde{M}_{i}$ and $\tilde{M}_{a}$ for sample A1 (almost a factor of 70), and the presence of large oscillations in $\tilde{\tau}_{i}$ when $\tilde{\tau}_{a}=0$ for sample A2, are compelling demonstrations that the isotropic dHvA magnetisation of a system can sometimes provide significantly more information than the anisotropic signal. The capability to measure both components with very high sensitivity gives our technique enormous potential for measurements of the $\mathrm{dHvA}$ effect when the Fermi surface, or certain pockets of the Fermi surface, are not strongly anisotropic, or when orientation suppresses the anisotropic response.

\section{SENSITIVITY AND CALIBRATION}

The calibration of our magnetometer was based on previous measurements of $\mathrm{Hg}_{1-x} \mathrm{Fe}_{x} \mathrm{Se} .{ }^{18}$ The Brillouin paramagnetism of $\mathrm{Fe}^{3+}$ in this system is known to have a saturation value of $5 \mu_{B}$ per ion, and it was, therefore, possible to calibrate the saturation magnetisation of the Brillouin response (shown as torque, rather than magnetisation in Fig. 4(c)) against this value. The error in our calibration is that involved in calculating the number of $\mathrm{Fe}^{3+}$ ions in our sample. The $\mathrm{Fe}^{3+}$ concentration was deduced from the measured dHvA frequency and multiplied by the volume of the sample to give the actual number of $\mathrm{Fe}^{3+}$ ions present. By far the dominant error in this calculation is the uncertainty in the dimensions of the sample, which, being small and somewhat irregular in shape, was difficult to measure precisely. We estimate the error involved in our calibration to be $\pm 15 \%$. The absolute values of torque and magnetisation given in Figs. 4 and 5 are, therefore, subject to this uncertainty.

To obtain values for the moment sensitivity of our magnetometer, we used the amplitude of the dHvA effect in the isotropic and anisotropic magnetisation of sample A1. We determined the amplitude of the smallest clearly resolvable torque oscillations to be $\sim 4 \times 10^{-14} \mathrm{~N} \mathrm{~m}$ at an applied field of $3.5 \mathrm{~T}$. This converts to a magnetic moment sensitivity of $4.4 \times 10^{-14} \mathrm{~J} / \mathrm{T}$ at $3.5 \mathrm{~T}$ for the isotropic magnetisation (this can also be seen from inset (ii) of Fig. 5(a)), and $1.1 \times 10^{-14}$ $\mathrm{J} / \mathrm{T}$ at $3.5 \mathrm{~T}$ for the anisotropic magnetisation. Both of these 
values are field dependent, and translate to a sensitivity at $30 \mathrm{~T}$ of $2.4 \times 10^{-14} \mathrm{~J} / \mathrm{T}$ for the isotropic magnetisation, and $1.3 \times 10^{-15} \mathrm{~J} / \mathrm{T}$ for the anisotropic magnetisation. These values have an experimental error of approximately $\pm 15 \%$.

We also calculated the expected sensitivity of the cantilever based on the relation between fractional resistance change of the piezoresistive path and longitudinal stress in a rectangular cantilever beam. ${ }^{30,33,34}$ Using $\pi_{L}=4.5$ $\times 10^{-10} \mathrm{~m}^{2} \mathrm{~N}$ as the longitudinal piezo coefficient for the cantilever material ${ }^{34}$ and following Ref. 30 to account for the shape of the cantilever, we obtained a "theoretical" calibration for the torque of $\tau=\Delta V /\left(2.5 \times 10^{6} V_{0}\right) \mathrm{N} \mathrm{m}$, where $\Delta V$ and $V_{0}$ are, respectively, the output and excitation voltages of our Wheatstone bridge circuit. The experimental calibration described above gives the equivalent expression $\tau=\Delta V /\left(5.2 \times 10^{6} V_{0}\right) \mathrm{N} \mathrm{m}$, so that theoretical and experimental calibrations agree within a factor of $\sim 2$. The experimental calibration, however, is more reliable due to uncertainties concerning, for example, the correct value of $\pi_{L}$ for our cantilever. ${ }^{33}$

\section{DISCUSSION AND PERSPECTIVES}

Torque measurements of anisotropic magnetisation using high sensitivity microcantilevers are widely performed, but sensitive measurement of the isotropic magnetisation of small samples, especially at millikelvin temperatures and in very high magnetic fields, is in general extremely difficult. The technique we describe here to exploit the sensitivity of microcantilevers for measurements of isotropic magnetisation is, therefore, a useful advance in this field, offering an improvement of several orders of magnitude on the sensitivities of commercial magnetometers. Commercial systems currently available have best sensitivities of $10^{-8}-10^{-11} \mathrm{~J} / \mathrm{T}$ for isotropic magnetisation ${ }^{35}$ and, in most cases, do not operate below $1.9 \mathrm{~K}$ or above $16 \mathrm{~T}$. More specialised systems to measure magnetisation to very low temperature ${ }^{17,36,37}$ or very high magnetic field ${ }^{17,18}$ have been reported, but the sensitivity of our magnetometer over a wide field range compares favourably in each case. The convenience and speed at which a measurement of both the isotropic and anisotropic magnetisation can be carried out are considerable additional advantages of our technique.

A number of ways to improve or extend the capabilities of our magnetometer are, however, identifiable. The most obvious is in improving the magnetic characteristics of the dysprosium ferromagnet. The polycrystalline dysprosium we used has magnetisation which is both lower than expected and dependent on the applied magnetic field throughout the whole range of our measurement (Fig. 3). A dysprosium single crystal, oriented with the applied field parallel to the easy axis of magnetisation, would have a fully saturated induction of $3.6 \mathrm{~T}$ at fields well below $1 \mathrm{~T}^{26} \mathrm{We}$ are now replacing our polycrystalline dysprosium with a single crystal of the same dimensions ${ }^{38}$ and expect to obtain an improved sensitivity of better than $1.8 \times 10^{-14} \mathrm{~J} / \mathrm{T}$ for the isotropic magnetisation, which is, moreover, independent of the applied magnetic field above $\sim 0.5 \mathrm{~T}$.
The small size of the piezoresistive cantilevers makes them difficult to calibrate accurately without using a test sample of known magnetisation, as we have done here. However, we envisage the greatest potential of our magnetometer to be in measurement of de Haas-van Alphen oscillations or other anomalous magnetic signatures such as phase transitions, where both isotropic and anisotropic components may contain considerable and complementary information, but where absolute values of the magnetisation are not necessary.

Our magnetometer is currently incorporated into a ${ }^{3} \mathrm{He}$ cryogenic system, but modification of the setup for implementation on a dilution refrigerator would be straightforward, especially where a rotation or translation mechanism is already in place. In this case, the limitation on the lowest operating temperature would be self-heating of the piezo cantilever due to the sensing current.

Finally, we comment that the method of using a moveable ferromagnet to create a local and "switchable" magnetic field gradient could be adapted in a number of ways to develop similar systems for different cantilever techniques or larger samples. However, there are limitations on the feasible sample size due to both the smaller field gradient produced and the larger forces experienced by the ferromagnet when its size is increased.

\section{CONCLUSIONS}

We have developed a simple technique for measuring the full, isotropic and anisotropic, magnetisation of very small bulk samples by combining existing piezoresistive microcantilever techniques with a small moveable bar of ferromagnetic dysprosium that can create a highly local field gradient of up to $4200 \mathrm{~T} / \mathrm{m}$ at the sample. Our magnetometer achieves sensitivities of $2.4 \times 10^{-14} \mathrm{~J} / \mathrm{T}$ for the isotropic magnetisation, and $1.3 \times 10^{-15} \mathrm{~J} / \mathrm{T}$ for the anisotropic magnetisation and is easy to construct and use. The system is ideal for use at millikelvin temperatures and very high magnetic fields, but is extremely versatile and can operate in temperatures up to $\sim 85 \mathrm{~K}$ and in magnetic fields as low as 1 or $2 \mathrm{~T}$.

\section{ACKNOWLEDGMENTS}

This work was supported by the Stichting Fundamenteel Onderzoek der Materie (FOM) with financial support from the Nederlandse Organisatie voor Wetenschappelijk Onderzoek (NWO).

${ }^{1}$ See, for example, the collection of review articles in J. Magn. Magn. Mater. Vol. 100 (1991).

${ }^{2}$ M. Bravin, S. A. J. Wiegers, P. Wolf, and L. Puech, J. Low Temp. Phys. 89, 723 (1992).

${ }^{3}$ D. Hautot, Q. A. Pankhurst, and J. Dobson, Rev. Sci. Instrum. 76, 045101 (2005).

${ }^{4}$ S. Foner, J. Appl. Phys. 79, 4740 (1996).

${ }^{5}$ L. DiMichele, C. Shelly, J. Gallop, and O. Kazakova, J. Appl. Phys. 108, 103918 (2010).

${ }^{6}$ C. Rossel, P. Bauer, D. Zech, J. Hofer, M. Willemin, and H. Keller, J. Appl. Phys. 79, 8166 (1996).

${ }^{7}$ E. Nazaretski, E. A. Akhadov, I. Martin, D. V. Pelekhov, P. C. Hammel, and R. Movshovich, Appl. Phys. Lett. 92, 214104 (2008). 
${ }^{8}$ N. E. Jenkins, L. P. DeFlores, J. Allen, T. N. Ng, S. R. Garner, S Kuehn, J. M. Dawlaty, and J. A. Marohn, J. Vac. Sci. Technol. B 22, 909 (2004).

${ }^{9}$ S. A. J. Wiegers, A. S. van Steenbergen, M. E. Jeuken, M. Bravin, P. E. Wolf, G. Remenyi, J. A. A. J. Perenboom, and J. C. Maan, Rev. Sci. Instrum. 69, 2369 (1998).

${ }^{10}$ C. Lupien, B. Ellman, P. Grutter, and L. Taillefer, Appl. Phys. Lett. 74, 451 (1999).

${ }^{11}$ H. Shishido, A. F. Bangura, A. I. Coldea, S. Tonegawa, K. Hashimoto, S. Kasahara, P. M. C. Rourke, H. Ikeda, T. Terashima, R. Settai, Y. Onuki, D. Vignolles, C. Proust, B. Vignolle, A. McCollam, Y. Matsuda, T. Shibauchi, and A. Carrington, Phys. Rev. Lett. 104, 057008 (2010).

${ }^{12}$ J. G. E. Harris, D. D. Awschalom, F. Matsukura, H. Ohno, K. D. Maranowski, and A. C. Gossard, Appl. Phys. Lett. 75, 1140 (1999).

${ }^{13}$ M. Qvarford, K. Heeck, J. G. Lensink, R. J. Wijngaarden, and R. Griessen, Rev. Sci. Instrum. 63, 5726 (1992).

${ }^{14}$ I. M. A. Bominaar-Silkens, Ph.D. thesis, Radboud University Nijmegen, Netherlands (2010), and references therein.

${ }^{15}$ R. Nagendran, N. Thirumurugan, N. Chinnasamy, M. P. Janawadkar, and C. S. Sundar, Rev. Sci. Instrum. 82, 015109 (2011).

${ }^{16}$ B. C. Stipe, H. J. Mamin, T. D. Stowe, T. W. Kenny, and D. Rugar, Phys. Rev. Lett. 86, 2874 (2001).

${ }^{17}$ J. S. Brooks, M. J. Naughton, Y. P. Ma, P. M. Chaikin, and R. V. Chamberlin, Rev. Sci. Instrum. 58, 117 (1986).

${ }^{18}$ U. Zeitler, A. Wittlin, J. C. Maan, W. Dobrowolski, and A. Mycielski, Phys. Rev. B 54, 15258 (1996)

${ }^{19}$ D. Rugar, C. S. Yannoni, and J. A. Sidles, Nature (London) 360, 563 (1992).

${ }^{20}$ J. A. Sidles, J. L. Garbini, K. J. Bruland, D. Rugar, O. Züger, S. Hoen, and C. S. Yannoni, Rev. Mod. Phys. 67, 249 (1995).

${ }^{21}$ C. L. Degen, M. Poggio, H. J. Mamin, C. T. Rettner, and D. Rugar, Proc. Natl. Acad. Sci. U.S.A. 106, 1313 (2009).

${ }^{22}$ The field profile shown was calculated analytically following Ref. 23. Similar results were obtained from finite element calculations. The dimensions and geometry of the ferromagnet were chosen as optimal for our purpose after performing these calculations for several different geometries.

${ }^{23}$ R. Engel-Herbert and T. Hesjedal, J. Appl. Phys. 97, 074504 (2005).

${ }^{24}$ P. G. van Rhee, Masters thesis, Radboud University Nijmegen, Netherlands (2010).

${ }^{25}$ The effect of a varying $d B / d x$ across the sample has not been quantified, but measurements at different ferromagnet-sample separations show predictable variations in the signal amplitude, which suggest an average value of $d B / d x$ is an acceptable description of $\nabla B$ experienced by a typical $50 \mu \mathrm{m}$ thick sample.

${ }^{26}$ V. Stepankin, Physica B 211, 345 (1995).

${ }^{27}$ S. A. Nikitin, G. I. Kataev, V. P. Posyado, and G. E. Chuprikov, Cryogenics 18, 153 (1978).

${ }^{28}$ Dysprosium foil, $0.1 \mathrm{~mm}$ thick, 99.9\%, Alfa Aesar GmbH \& Co KG, Zeppelinstrasse 7, 76185 Karslruhe, Germany.

${ }^{29}$ Seiko Instruments Inc., 1-8 Nakase, Mihama-ku, Chiba 261-8507, Japan.

${ }^{30}$ H. Takahashi, K. Ando, and Y. Shirakawabe, Ultramicroscopy 91, 63 (2002).

${ }^{31} \mathrm{~A}$ different ferromagnetic material would allow this upper limit on temperature to be increased. For example, terbium has a Curie temperature of $219 \mathrm{~K}$ with a saturation induction of $\sim 3.3 \mathrm{~T}$, only slightly lower than that of dysprosium (see Ref. 27). Terbium, however, is considerably more expensive than dysprosium.

${ }^{32}$ I. M. Lifshitz and A. M. Kosevich, Sov. Phys. JETP 2, 636 (1956).

${ }^{33}$ C. Bergemann, Ph.D. thesis, University of Cambridge, UK (1999).

${ }^{34}$ E. Ohmichi and T. Osada, Rev. Sci. Instrum. 73, 3022 (2002).

${ }^{35}$ For example, Quantum Design, Inc. San Diego, CA 92121-3733, USA: PPMS and MPMS with various options. Lake Shore Cryotronics, Inc., Westerville, OH 43082, USA: 7400 series VSM.

${ }^{36}$ S. Legl, C. Pfleiderer, and K. Kramer, Rev. Sci. Instrum. 81, 043911 (2010).

${ }^{37}$ A. Morello, W. G. J. Angenent, G. Frossati, and L. J. de Jongh, Rev. Sci. Instrum. 76, 023902 (2005).

${ }^{38}$ Dy single crystal, Materials Preparation Center, Ames Laboratory, US DOE Basic Energy Sciences, Ames, IA, USA. 